\title{
Möglichkeiten und Grenzen der Selbsthilfeförderung in Indonesien
}

Die Hoffnungen der Modernisierungstheoretiker der 50er Jahre, Entwicklung in der Dritten Welt durch die Umwandlung «traditioneller» Gesellschaften in «moderne» Gesellschaften nach westlichem Vorbild zu erreichen, konnten nicht erfüllt werden, da die erhofften Sikkereffekte der wirtschaftlichen Entwicklung, welche die ärmeren Bevölkerungsschichten hätten erreichen sollen, ausgeblieben sind. Auch die damals existierenden Ansätze zur Förderung lokaler Dorfgemeinschaften, die unter dem Begriff "community development» bekannt wurden, haben bezüglich der Förderung und Besserstellung marginalisierter Bevölkerungsgruppen ihre Ziele weitgehend verfehlt. Einer der Hauptgründe dafür war unter anderen die mangelnde Stratifikation der Zielgruppen, die allzu oft dazu führte, daß sich die dörflichen Eliten und die traditionellen Führer zusammentaten und von den Entwicklungsprojekten am meisten profitierten. Somit trat der erhoffte Abbau der Disparitäten zwischen den einzelnen Schichten innerhalb der Dorfgemeinschaften nicht ein, und die auf traditionellen Strukturen beruhenden Ungleichgewichte wurden perpetuiert oder sogar noch verschlimmert. Diese Erfahrungen haben Anfang der 80er Jahre zur Einsicht geführt, daß - falls mit einer armutsorientierten Entwicklungsstrategie die Basis erreicht werden soll - innerhalb von Dorfgemeinschaften weitere Stratifikationen wie beispielsweise Handwerker, Händler, Frauen, Bauern usw. vorgenommen werden müssen, um damit die Zielgruppe zu erkennen, welche der Hilfe am meisten bedarf. Aufgrund dieser Erkenntnis entstanden unter dem Begriff des "Selbsthilfeansatzes» (der "Hilfe zur Selbsthilfe» propagiert) neue, armutsorientierte, partizipative Entwicklungskonzepte mit Programmen auf der Mikroebene und "Bottom up»-Planungsverfahren.

\section{Der Selbsthilfeansatz}

Der Selbsthilfeansatz kann als Synthese früherer Entwicklungsstrategien verstanden werden und vereint diverse, bereits in anderen Konzepten verwendete Entwicklungselemente und -aspekte wie beispielsweise "small is beautiful», "Entwicklung von unten», «self-reliance», «soziale Entwicklung», «zielgruppenorientierte Entwicklung» oder «angepaßte Technologie». Der Selbsthilfeansatz behandelt aber vorwiegend ein Element der Armutsbekämpfung: die Förderung der Selbsthilfe der Armen. Im Gegensatz zu seinen Vorgängern be- rücksichtigt der Selbsthilfeansatz aber vermehrt die soziale Schichtung einer Gesellschaft oder Gemeinschaft. Die Anpassung von Projekten an lokale Gegebenheiten ist dabei von größter Relevanz. Zentrales Anliegen der Hilfe zur Selbsthilfe ist die Bildung produktiver, selbständig funktionierender, lokaler Ökonomien als Voraussetzung nachhaltiger gesellschaftlicher Entwicklung (KORTEN 1989: S. 13). Hilfe zur Selbsthilfe soll der Armutsbevölkerung vermehrt den Zugang sowohl zu zusätzlichen immateriellen Ressourcen (wie z. B. Know-how) als auch zu anderen Ressourcen wie Krediten, Rohstoffen, Land, Wasser usw. ermöglichen (VERHAGEN 1987: S. 15).

Zu den zentralen Komponenten des Selbsthilfeansatzes gehören die Selbsthilfeförderungsinstitutionen, die Hilfe zur Selbsthilfe in die Praxis umsetzen. Dabei wird die These vertreten, daß regierungsunabhängige Entwicklungsorganisationen $(\mathrm{NGO}=$ Non-Governmental Organization)' als Selbsthilfeförderungsinstitutionen effektiver und effizienter als staatliche Entwicklungsinstitutionen Aufgaben im Bereich der Selbsthilfeförderung übernehmen könnten, weil sie sowohl die ideologische Grundhaltung als auch die institutionelle Kapazität besitzen, um die Bedürfnisse der Ärmsten zu berücksichtigen und in ihre Projekte zu integrieren. Der Grundgedanke der Hilfe zur Selbsthilfe ist folgender: Durch die Initiierung und Förderung von Selbsthilfe entstehen positive Rückkoppelungseffekte innerhalb der Zielgruppe, welche wirtschaftliche, politische und soziokulturelle Entwicklungen auslösen, die im Endeffekt zu vermehrter Selbständigkeit, zur Verbesserung der Lebensbedingungen und Lebenserwartung sowie zu einer angemessenen Form von Wohlstand innerhalb der Zielgruppe führen. Weiter wird angenommen, daß eine von ihrer Selbsthilfeförderungsinstitution finanziell und strukturell unabhängig gewordene Selbsthilfeorganisation oder -gruppe selbst die Rolle einer solchen Institution übernehmen und ihrerseits neue Selbsthilfevereinigungen gründen würde. Die damit überflüssig gewordene "Initial-Selbsthilfeförderungsinstitution» könnte sich nach erfüllter Aufgabe zurückziehen und ihre Aktivitäten in andere geographische Gebiete verlegen und dort ihre Dienste anbieten. In diesem Szenario würde die Selbsthilfe nach einer gewissen Zeit lokal bzw. regional selbsttragend.

Yves Guinand, dipl. Geograph, c/o Dr. Ekow Akyeampong, Project ICRAF/ISABU, B. P. 173, Gitega, Burundi 
Der Selbsthilfeansatz postuliert somit eine Bewegungsbildung marginalisierter Bevölkerungsschichten von unten.

\section{Die indonesischen Selbsthilfeförderungsinstitutionen Bina Swadaya und Lembaga Studi Pembangunan}

Bina Swadaya gilt heute in Indonesien als die größte unabhängig arbeitende Entwicklungsorganisation. Die Vorläufer von Bina Swadaya, zu denen verschiedene Bauernverbände und -organisationen zählen, gehen bis ins Jahr 1958 zurück. Aus der 1967 gegründeten Yayasan Sosial Tani Membangun (YSTM = soziale Stiftung zur Entwicklungsförderung der Bauern) ging 1981 die Selbsthilfeförderungsinstitution Bina Swadaya hervor.

Ziel von Bina Swadaya ist die Überwindung der Armut durch eine Verbesserung sozioökonomischer Lebensbedingungen unter den marginalisierten Bevölkerungsschichten. Diese soll mit Hilfe geeigneter Maßnahmen wie der Selbsthilfeförderung erreicht werden (vgl. BINA SWADAYA 1986: S. 4). Bina Swadaya konzentriert sich auf die ländliche Entwicklung und die Verbesserung wirtschaftlicher sowie sozialer Strukturen und fördert Selbsthilfeorganisationen, welche in Indonesien als «Pre-Cooperatives» oder "Usaha Bersama» (UB = Gemeinschaftsaktion) bezeichnet werden. "Pre-Cooperatives» oder "Usaha Bersama» sind informelle Selbsthilfegruppen ohne feste Organisationsstrukturen und ohne formelle amtliche Registrierung. Sie sind in Indonesien als Übergangslösung verfassungsrechtlich statthaft. Bina Swadaya nennt seine Selbsthilfegruppen deshalb «Usaha Bersama» (und nicht «Pre-Cooperatives»), weil das Wort «koperasi» bei der Dorfbevölkerung negative Erfahrungen in Erinnerung ruft, die sie mit staatlichen Kooperativen hatte machen müssen (vgl. ELDRIDGE 1989: S. 24 ff.). Die Organisation sieht sich eher als dienstleistungsorientierte Entwicklungsinstitution und weniger als Wegbereiter eines soziopolitischen Wandels der indonesischen Gesellschaft von unten: Bina Swadaya versteht sich als Motivator, Kommunikator und Fazilitator ihrer Selbsthilfegruppen (vgl. BONGARTZ 1987: S. 72 und ELDRIDGE 1989: S. 29).

Lembaga Studi Pembangunan (LSP = Institut für Entwicklungsstudien) ist ein typisches Beispiel einer von indonesischen Intellektuellen der städtischen Mittelklasse mit Erfahrungen aus der Privatwirtschaft und aus Regierungsinstitutionen Mitte der 70er Jahre gegründeten, unabhängigen Entwicklungsorganisation. Anfänglich arbeitete LSP als PR-Agentur mit dem Ziel, die Entwicklungsprobleme Indonesiens zu studieren und durch gezielte Öffentlichkeitsarbeit (Verbreitung von Studien und Zeitschriften, Organisation von Workshops und informellen Meetings mit Regierungsvertretern usw.) einem breiteren Publikum bekannt zu machen. Damit wollte sie hauptsächlich Regierungsangehörige, die städtische Oberschicht sowie Vertreter und Experten ausländischer Entwicklungshilfeorganisationen sensibilisieren und für ihre Anliegen gewinnen. 1979 entschloß sich LSP, als Selbsthilfeförderungsinstitution die eigenen Ideen und Konzepte in die Tat umzusetzen. Vorerst arbeitete LSP mit anderen, bereits bestehenden Selbsthilfeförderungsinstitutionen zusammen, um so Erfahrungen zu sammeln. Ihre Projekte hatten vorwiegend Pilotcharakter und dienten der partizipativen, aktionsorientierten Forschung.

Entwicklungsverständnis und -ziele beinhalten bei LSP nicht nur wirtschaftliche und soziale Förderungsmaßnahmen. Die Organisation verfolgt mit ihren Konzepten und Ideen auch gesellschaftspolitische Ziele und will Veränderungen in der indonesischen Gesellschaftsstruktur bewirken. Sie möchte als "agent of change» der indonesischen Gesellschaft verstanden werden und stellt den Anspruch, den Prozeß des sozialen Wandels von unten einleiten und fördern zu wollen. Die Entwicklungsschwerpunkte liegen im städtischen informellen Sektor und im beschäftigungspolitischen Bereich. Die Bildung von Kooperativen dient als Instrument zur Umverteilung von Ressourcen zugunsten wirtschaftlich schwächerer Gruppen, die dadurch mehr Selbständigkeit erhalten sollen. Der Verfassungsartikel Nr. 33 von 1945, welcher die gemeinsame Nutzung nationaler Ressourcen ausdrücklich befürwortet, legitimiert ein solches Vorgehen. LSP fördert Selbsthilfeorganisationen, d. h. "Pre-Cooperatives», welche später den Status registrierter Kooperativen erlangen sollen.

Wie Bina Swadaya versteht sich LSP als Motivator, Kommunikator und Fazilitator ihrer Selbsthilfegruppen (vgl. BONGARTZ 1987: S. 72 und ELDRIDGE 1989: S. 29). Heute ist auch LSP, obwohl in der Größe (Anzahl Mitarbeiter, Anzahl laufender Projekte, Finanzvolumen usw.) nicht mit Bina Swadaya vergleichbar, zu einer der größten Selbsthilfeförderungsinstitutionen Indonesiens angewachsen (vgl. ELDRIDGE 1989: S. 27 und SASONO 1988: S. 11).

\section{Zentrale Aspekte der Selbsthilfeförderung in Indonesien}

Im indonesischen Sprachgebrauch hat der Ausdruck "Non-Governmental Organization" die Konnotation von «regierungsfeindlich». Für diese Assoziation sind historische Gründe verantwortlich. Während der revolutionären Periode (1945-49) bedeuteten die Begriffe «koperasi» „Kooperation mit der Kolonialmacht“ und «nonkoperasi” „Widerstand gegen die niederländische Kolonialmacht". In Indonesien sind bis heute die Befürchtungen bestehen geblieben, daß "non-governmental» als «regierungsfeindlich» aufgefaßt werden könnte (vgl. ISMAWAN 1987: S. 1). Deshalb wurde der Ausdruck "NonGovernmental Organization" durch einen Begriff ersetzt, der die Funktion von NGOs im Kontext des Entwicklungsprozesses genauer wiedergibt. NGOs werden in Indonesien als LPSM bezeichnet (Lembaga Pengembangan Swadaya Masyarakat = Organisation zur Entwicklung und Förderung von Selbsthilfegruppen, oder kürzer: Selbsthilfeförderungsinstitution). LPSM unter- 
stützen und fördern kleine, meistens auf Dorfebene operierende Selbsthilfegruppen, die unter dem Begriff LSM (Lembaga Swadaya Masyarakat $=$ Selbsthilfeorganisation) zusammengefaßt sind (vgl. KUSUMAHADI 1987: S. 11 f. und ELDRIDGE 1989: S. 3 f.).

Die Gründung und der Aufbau von Selbsthilfegruppen auf nationaler, regionaler und lokaler Ebene gehören für viele indonesische NGOs zum zentralen Aufgabenbereich. Der Entschluß zum Beitritt in eine Selbsthilfegruppe erfolgt vor allem aus ökonomischen und soziokulturellen Beweggründen. Viele Selbsthilfegruppen stellen ihren Mitgliedern Kreditsysteme zur Verfügung und helfen ihnen beim Aufbau und bei der Ausübung ökonomischer Aktivitäten. Mitglied in einer oder mehreren Selbsthilfegruppen zu sein, ist für die betreffende Person oder Familie zudem mit Prestigegewinn verbunden.

Der Aufbau eines Kreditsystems (simpan pinjam = Sparund Kreditaktivitäten) ist die weitaus häufigste Art, Ressourcen innerhalb der Selbsthilfegruppe zu mobilisieren. Grund dafür ist die vergleichsweise einfache Implementierung und Überwachung solcher Aktivitäten. Der Aufbau organisatorischer Strukturen, welche die Akkumulierung und Verwaltung von Ersparnissen ermöglichen, gehört zur primären Aufgabe einer Selbsthilfegruppe. Damit soll für die Mitglieder die Unabhängigkeit von informellen Geldverleihern und später der Zugang zum regulären Bankensystem erreicht werden. Die meisten Selbsthilfegruppen versuchen, ihre eigenen Kapitalbildungsformen zu organisieren, die es ihnen erlauben, regelmäßig Kleinbeträge zu sparen und Kredite aus dem Gruppenkapital zu beziehen. In den von Bina Swadaya unterstützten Selbsthilfegruppen können in der Regel die folgenden vier verschiedenen Sparformen unterschieden werden (IFDA 1989: S. 20 ff.):

1. Jedes Mitglied muß periodisch (wöchentlich oder monatlich) einen bestimmten obligatorischen Sparbetrag (tabungan wajib) an seine Selbsthilfegruppe bezahlen, welcher, solange man Mitglied bleibt, nicht abgehoben werden darf. Dieser Sparbetrag variiert zwischen Rp. 100.- und Rp. 500.- (US-\$0.10 und 0.40).'

2. Jedes Mitglied kann freiwillige Sparbeiträge (tabungan sukarela) leisten, welche nachher jedem Mitglied als Kredit vergeben werden können. Die Zinsrate beträgt monatlich 2,5\% und wird auf alle Mitglieder gleichermaßen verteilt.

3. Ein Solidaritätssparbeitrag (tabungan setia kawan) wird direkt auf ein Sparkonto von Bina Swadaya deponiert. Er trägt annähernd dieselben Zinsen, wie sie von staatlichen Banken bezahlt werden. Mit Hilfe des durch Bina Swadaya zur Verfügung gestellten Solidaritätsspar- und Kreditsystems können die Selbsthilfegruppen neben den selbstverwalteten Sparguthaben zusätzlich Geld auf einem von Bina Swadaya verwalteten speziellen Bankkonto anlegen. Von diesem Solidaritätsguthaben (tabungan setia kawan) kann jede beteiligte Gruppe Solidaritätskredite (kredit setia kawan) in Anspruch nehmen (VERHAGEN 1987: S. 69).
4. Beim Eintritt in eine Selbsthilfegruppe hat jedes Neumitglied einen einmaligen Eintrittsbeitrag (tabungan pokok) zu leisten. Dieser liegt je nach Gruppe zwischen Rp. 1000.- und Rp. 10 000.- (US-\$ 0.80 und 8.-).

Neben den Spar- und Kreditaktivitäten werden die Selbsthilfegruppenmitglieder zum Aufbau ökonomischer Aktivitäten motiviert und unterstützt. Die beiden vom Verfasser untersuchten Selbsthilfeförderungsinstitutionen Bina Swadaya und LSP bieten verschiedene Schulungs- und Weiterbildungskurse für ihre Selbsthilfegruppenmitglieder an. ${ }^{3}$

\section{Rahmenbedingungen regierungsunabhängiger Selbsthilfeorganisationen beeinflussen die praktische Umsetzung des Selbsthilfeansatzes in Indonesien}

Die wirtschaftliche Rezession wurde durch den Zerfall der Erdölpreise (Indonesien ist nach China der zweitgrößte Erdölproduzent im ostasiatischen Raum) und die Mißerfolge zahlreicher wachstums- und einkommensfördernder Maßnahmen Anfang der 80er Jahre eingeleitet. Zusätzlich hat Indonesien mit Problemen des Bevölkerungswachstums und der Landverknappung, vor allem auf den bevölkerungsreichen sogenannten inneren Inseln (Java, Madura, Bali und West-Lombok), zu kämpfen. Dies läßt den ohnehin schon überdurchschnittlich hohen Anteil unterbeschäftigter und arbeitsloser Indonesier weiter ansteigen.

Die Wirtschaftskrise und die damit verbundene Limitierung staatlicher Mittel zugunsten von Entwicklungsprojekten sowie das durch verschiedene Mißerfolge genährte Mißtrauen ausländischer Geldgeber gegenüber staatlichen Entwicklungsinstitutionen haben den Aufschwung privater, regierungsunabhängiger Entwicklungsorganisationen begünstigt. So entstanden in den 80er Jahren zahlreiche regierungsunabhängige Selbsthilfeorganisationen. Diese haben bei der Implementierung ihrer Selbsthilfeprogramme und -projekte mit verschiedenen Problemen zu kämpfen, deren Ursachen vielfach in den politischen und soziokulturellen Rahmenbedingungen begründet sind, unter denen Entwicklungspolitik in Indonesien stattfindet.

Politische und soziokulturelle Rahmenbedingungen sowie das indonesische Gesellschaftssystem beeinflussen Selbsthilfeorganisationen und deren Aktivitäten aufvielfältigste Weise. Offiziell ist Indonesien zwar ein moderner Staat mit demokratischen Entscheidungsinstrumentarien. De facto handelt es sich aber um ein oppositionsloses politisches System, dessen formale Strukturen eher der Legitimation als der politischen Willensbildung dienen. In diesem autoritären politischen System, in dem das Militär neben der Landesverteidigung in allen gesellschaftlichen Bereichen entscheidenden Einfluß nimmt, ${ }^{4}$ liegt die politische Entscheidungsgewalt seit dem Staatsstreich von 1965/66 (bei dem General Suharto sich an der Spitze des Staates etablierte) fest in den Händen einer 
kleinen Elite von Militärs und Technokraten. Seit der Machtübernahme durch Suharto ist es dem Regime mit geschickten taktischen Manövern gelungen, die politische Opposition in die Bedeutungslosigkeit zu verdrängen und somit alle politischen und wirtschaftlichen Aktionen zu beeinflussen oder zu kontrollieren.

Die entwicklungspolitischen Entscheidungen und Richtlinien werden in Indonesien von der Regierung in Form von Fünfjahresentwicklungsplänen (Repelita) nach modernisierungstheoretischem Grundmuster ausgearbeitet und implementiert. Das gut ausgebaute staatliche Kontrollsystem reicht bis auf die Dorfebene, wo die LKMD (Lembaga Ketahanan Masyarakat Desa = Organisation zur Aufrechterhaltung von Sicherheit und Ordnung auf Dorfebene) als Koordinatoren dörflicher Aktivitäten (Sicherheit, wirtschaftliche Aktivitäten, Infrastrukturausbau, kulturelle Veranstaltungen usw.) Gruppen und Organisationen - gleichgültig, ob staatlich oder regierungsunabhängig, ob formell oder informell - kontrollieren und beeinflussen. Für ihre Aktionen auf Dorfebene sind Selbsthilfeförderungsinstitutionen und informelle Selbsthilfegruppen auf das Einverständnis oder mindestens auf die Toleranz z. B. des betreffenden Distriktvorstehers (Bupati) angewiesen.

Als Anfang der 80er Jahre NGOs an Einfluß gewannen und verschiedene unter ihnen erfolgreiche Selbsthilfeprojekte vorweisen konnten, verabschiedete die Regierung 1985 ein Gesetz, welches unter anderem auch sozial engagierte Organisationen in ihren Aktivitäten hätte einschränken sollen. Dieses Gesetz legt genau fest, auf welche Art, in welchem Umfang und in welchen Bereichen NGOs am nationalen Entwicklungsprozeß teilhaben dürfen. Folgende 1983 von Emil Salim, dem Minister für Umwelt- und Bevölkerungsfragen, gemachte Aussage wurde 1985 in dieses Gesetz integriert: "As long as the differences only concern problems of implementation, then the LSMs' (Selbsthilfeorganisationen) right to exist is guaranteed. But if the differences concern more 'philosophical' matters, like differences in ideology or differences in national aims, then clearly any LSM with those sorts of differences with the government will not have the right to exist" (SALIM 1984: S. 71). Die Regierung duldet von NGOs im allgemeinen als einziges soziales Tätigkeitsfeld die Gründung und Förderung von Kooperativen (vgl. SASONO 1988: S. 43).

De jure ist das Gesetz repressiv, denn es versucht, die Bewegungsfreiheit indonesischer NGOs einzuschränken. Aber in der Praxis ist es wohl doch eher so, daß die schwerfällige Bürokratie und die relative Unüberblickbarkeit der vielen verschiedenen Organisationen eine erfolgreiche Implementierung des Gesetzes und eine effiziente Kontrolle der NGOs verhindern. Zusätzliche Implementierungshemmnisse ergaben sich aufgrund der erwähnten schwierigen Wirtschaftsbedingungen, deretwegen das Entwicklungsbudget drastisch gekürzt werden mußte. NGOs sind seit Beginn des fünften Fünfjahresentwicklungsplans (Repelita V: 1984-1989) auch de jure als Partner im nationalen Entwicklungsproze $ß$ anerkannt, nicht nur, weil sie der Regierung als Konsulenten bei nationalen Regierungsprojekten und -programmen willkommen sind, sondern auch wegen der ausländischen Hilfsgelder, die NGOs zufließen.

Neben dem restriktiven politischen System wird die indonesische - und insbesondere die javanische - Gesellschaft von einem Normen- und Wertesystem bestimmt, das auf Ruhe, Ordnung und Einheit beruht und dessen wichtigste Komponenten (Kompromißbereitschaft sowie Konsensfindung) das Zusammenleben innerhalb ejner Gemeinschaft bestimmen. Die Ordnung eines Dorfsystems beruht auf dem Prinzip gemeinsam akzeptierter Entscheidungen, die durch einen Kompromiß aller vorgebrachten Gesichtspunkte zustande kommen. Der angestrebten Konsensfindung (mufakat) gehen in der Regel lange Diskussionen voraus (musyawarah). Im javanischen Ordnungssystem sind "musyawarah" und "mufakat" Voraussetzungen für ein harmonisches Zusammenleben. Idealisiert ist damit gemeint, daß Entscheidungen und Beschlüsse über den Konsens in harmonischer Übereinstimmung getroffen werden (vgl. BIJLMER und REURINK 1986: S. 144).

Die Bindung der Menschen an die Gemeinschaft ist religiös-magischer Natur und hängt mit einem mystisch bestimmten Weltbild zusammen, in dem das Dorfterritorium und die darin Lebenden als Pars pro toto den Kosmos widerspiegeln: Alles hängt mit allem zusammen. Das althergebrachte System von spontaner gegenseitiger Hilfsbereitschaft ist in den indonesischen Dorfgemeinschaften tief verwurzelt und zum Sinnbild des indonesischen Ordnungssystems geworden. Diese kommunale und gegenseitige Hilfeleistung oder Kooperation innerhalb einer Gemeinschaft wird mit dem Begriff "gotong royong ${ }^{5}$ angedeutet (vgl. ROEDER 1979: S. 191 und WAN. DELT 1989: S. 252). Die gesellschaftlichen Beziehungen sind in diesem System strikt hierarchisch geordnet und vertikal orientiert. Vertikale Beziehungen innerhalb der hierarchischen Strukturen sind auf einem patriarchalischen Patron-Klientel-Verhältnis aufgebaut. Persönlichkeiten, denen offiziell oder inoffiziell ein hoher sozialer Status attestiert wird, verfügen über nahezu uneingeschränkte Macht und Integrität und entziehen sich faktisch jeglicher Kritik und Kontrolle. Designierte Führer verpflichten sich ihrerseits, die Verantwortung für das Wohlergehen ihrer Untergebenen zu übernehmen. Letztere werden angehalten, sich ihrem Führer gegenüber loyal zu verhalten und ihn zu respektieren. Aus diesem hierarchischen sozialen Beziehungsgefüge heraus ist auch das Verhalten der Untergebenen zu verstehen, die akzeptieren, was von einer sozial höher gestellten Person gesagt oder befohlen wird. Durch dieses Verhalten entziehen sie sich andererseits der sozialen Verantwortung, Eigenentscheidungen zu treffen.

Dieses traditionelle Normen- und Wertesystem zerfällt heute jedoch langsam angesichts des schnellen Wandels, dem auch die indonesische Gesellschaft ausgesetzt ist. Vor allem in den urbanen Zentren haben moderne, westliche Werte an Einfluß gewonnen. Aber auch im ländlichen Raum werden Entscheidungen nicht mehr allein 
auf der Basis von "musyawarah untuk mufakat" (= Diskussion zum Zwecke der Konsensfindung) gefällt. Zudem muß man sich bewußt sein, daß dieses Normen- und Wertesystem schon immer nur in seiner idealisierten Form weitergegeben wurde und wohl eher einen Mythos darstellt als der Realität entspricht (vgl. BIJLMER und REU. RINK 1986: S. 144).

\section{Interne institutionelle und instrumentelle Probleme der Selbsthilfeförderungsinstitutionen als Ursache inadäquater Umsetzungspraktiken des Selbsthilfeansatzes}

Neben den oben erwähnten Rahmenbedingungen treten eine ganze Reihe interner Hemmnisse auf, die eine adäquate Umsetzung des Selbsthilfeansatzes in Indonesien erschweren. Vielfach mangelt es an organisatorischem Know-how und gut geschultem Personal. Diese institutionellen Probleme tragen dazu bei, daß Selbsthilfeinstrumente wie beispielsweise Zielgruppendefinition, partizipative Forschung und Planung, Aus- und Weiterbildung von Mitarbeitern und Selbsthilfegruppenmitgliedern usw. falsch interpretiert und deshalb weder richtig noch konsequent eingesetzt werden.

Engagement und Spontaneität boten zu Beginn bei beiden untersuchten Organisationen die Garantie für die Erhaltung eines gesunden Innovationspotentials, welches zu einer Reihe von äußerst erfolgreichen Projekten geführt hat. Diese haben die Aufmerksamkeit potentieller ausländischer Geldgeber auf sich gezogen, welche nach den Mißerfolgen mit staatlichen Entwicklungsinstitutionen gerne bereit waren, finanzielle Unterstützung zu gewähren. Der ausländische Geldfluß hat das Wachstum beider NGOs entscheidend beschleunigt und sie zum Ausbau ihres Büroapparates gezwungen. Diese Entwicklung birgt Gefahren in sich, denn jedes einzelne von NGOs aufgebaute Projekt hat seinen individuellen Charakter, d. h. es ist und bleibt ein Pilotprojekt, das nur begrenzt übertragbar ist. Der Pilotcharakter jedes einzelnen Projektes ist denn auch eines der Erfolgsgeheimnisse der Selbsthilfeförderung. Wenn nun Geldgeber NGOs damit beauftragen, ihre Pilotprojekte auszuweiten, d. h. dasselbe Projekt gleich mehrmals in verschiedenen Regionen zu kopieren, dann werden sie fehlender engagierter Mitarbeiter wegen nicht in der Lage sein, diesen Wunsch nachhaltig umzusetzen. Mit der schematischen Duplizierung von Projekten nimmt deren Erfolg ab, und der Verwaltungsaufwand der Organisation steigt überproportional an. Es müssen komplizierte administrative Strukturen geschaffen werden, und Aktivisten, die urspünglich in ihren Dörfern engagiert angefangen hatten, wirken später in Jakarta, im Hauptsitz ihrer Organisation, weit weg von der Basis. Im Grunde genommen sind aber nicht die Selbsthilfeförderungsaktivisten für eine solche Entwicklung verantwortlich zu machen, sondern vielmehr die Geldgeber.

Viele Selbsthilfeförderungsinstitutionen, insbesondere Bina Swadaya und LSP, sind in den letzten zehn Jahren so rasch gewachsen, daß sie die ursprünglichen Ziele der Selbsthilfeförderung je länger je mehr vernachlässigt haben und, gewollt oder ungewollt, ihr Image von einer entwicklungsorientierten Selbsthilfeorganisation hin zu einer profitorientierten Geschäftsstelle verändert haben. Die Organisationsstrukturen sind hierarchisch, bürokratisch und patriarchalisch geworden und unterscheiden sich heute nur noch geringfügig von denen staatlicher Entwicklungsinstitutionen. Dabei spielt die hierarchisch und patriarchalisch aufgebaute javanische Gesellschaftsstruktur eine gewichtige Rolle. Dazu beigetragen hat wohl auch die Annäherung beider Organisationen an staatliche Entwicklungsinstitutionen, von denen Teilprojekte in Regie übernommen wurden, welche von den Zielen sowie dem Selbstverständnis der Selbsthilfeförderung weit entfernt sind.

Ganz allgemein hängen Erfolg und Mißerfolg von Projekten noch immer stark von Einzelpersonen, wie z. B. den Gründern der Organisationen, ab. Die Aus- und Weiterbildung, insbesondere der Feldmitarbeiter, ist ungenügend. Ihr sozialer Status entspricht in beiden Organisationen nicht den Ansprüchen der Selbsthilfeförderung. Die Lohn- und Personalpolitik ist diesbezüglich ebenfalls nicht optimal gestaltet. NGOs sind vor allem im Felde auf kompetente Mitarbeiter angewiesen. Um aber solche nicht nur anzustellen, sondern auch zu behalten, bedarf es finanzieller wie auch institutioneller Anreize. Folgende Aussage über den sozialen Status von NGO-Feldmitarbeitern entspricht - von wenigen Ausnahmen abgesehen - auch den Verhältnissen bei Bina Swadaya und LSP: "In reality, however, they are (with the exception of highly specialized experts) - on the average - the youngest, least experienced, least educated, worst paid, etc. staff of NGOs" (BONGARTZ/KUSUMAHADI 1988: S. 20). LSP ist sich dieser Situation bewußt geworden und hat in Pabelan (Zentraljava) mit dem Aufbau eines speziellen Ausbildungszentrums für künftige NGO-Funktionäre die folgerichtige Maßnahme zur Beseitigung von Problemen im Zusammenhang mit dem Ausbildungsniveau des NGO-Personals, insbesondere der Feldmitarbeiter, ergriffen. Ende der 80er Jahre waren die Angestellten beider Organisationen jedoch noch nicht ihren Aufgaben und Tätigkeitsbereichen entsprechend ausgebildet. Doch sind Verbesserungen in diesem Bereich für die 90er Jahre in die Wege geleitet worden. Ein weiteres Dilemma ist im Zusammenhang mit den hierarchischen und patriarchalischen Organisationsstrukturen beider Organisationen zu sehen: Wie sollen Mitarbeiter von Selbsthilfeförderungsinstitutionen "partizipatorisch" Selbsthilfe fördern, wenn sie selbst in hierarchisch und patriarchalisch organisierten Institutionen tätig sind?

Die Finanzmittelbeschaffung zum Zwecke der Selbsthilfeförderung ist ein anderes Dilemma, mit dem sich die meisten indonesischen NGOs konfrontiert sehen. Zentrale Frage dabei ist, wie NGOs sich die nötigen Mittel beschaffen können, ohne in finanzielle Abhängigkeit der Geber zu geraten und ohne ihr Selbsthilfepotential (Ideologie, Idealismus, Strategie usw.) zu schwächen oder gar 
zu zerstören. Eine mögliche Strategie ist der Aufbau und die Förderung eigener wirtschaftlicher Produktionsbetriebe, welche die erwirtschafteten Profite den Selbsthilfeförderungsprogrammen und -projekten der Organisation überweisen können. Diese Idee ist begrüßenswert, doch die Praxis bei Bina Swadaya hat gezeigt, daß Gewinne und Profite der Produktionsbetriebe nicht in die Selbsthilfeförderung, sondern in die Produktionsbetriebe reinvestiert werden. Die Selbsthilfeförderungsabteilung von Bina Swadaya (PUSBINUB) ist somit trotz eigener Produktionsbetriebe weiterhin praktisch vollständig auf Fremdmittel angewiesen.

Eines der wichtigsten Arbeitsinstrumente in der Selbsthilfeförderung ist die Stratifikation und Identifikation der anvisierten Zielbevölkerung. Eine unsaubere Stratifikation und Identifikation kann erstens zu teilweise elitären, undemokratischen und von oben diktierten Programm- und Projektansätzen führen, zweitens dazu, daß marginalisierte Bevölkerungsschichten nur bedingt Zugang zu Selbsthilfegruppen finden, und drittens, daß die Führungspositionen innerhalb der Selbsthilfegruppen fast ausschließlich von der Dorfelite bekleidet werden.

Bei der Implementierung von Programmen und Projekten erweist es sich jedoch oft, daß eine klare Abgrenzung der Zielgruppe äußerst schwierig ist. Es ist z. B. nahezu ausgeschlossen, die Ärmsten der Armen, die völlig Mittellosen, in Projekte mit einzubeziehen, da sie keinen festen Aufenthaltsort haben und deshalb gar nicht oder nur äußerst schwer zu registrieren, zu organisieren und an einem bestimmten Ort festzuhalten sind. Um eine gewisse Erfolgsquote bei Projekten zu erzielen, konzentrieren sich Selbsthilfeförderungsinstitutionen wie LSP mehr und Bina Swadaya vor allem auf solche Menschen, die sich bereits über Eigenbesitz ausweisen und demnach auch etwas zu verlieren haben, was heißt, daß letztere daraufbedacht sind, bereits Erreichtes zu wahren oder zu mehren. Die fehlenden Erfolgsaussichten sowie das zu hohe Risiko veranlassen kaum eine Selbsthilfeförderungsinstitution, eine Zusammenarbeit mit den Ärmsten der Armen ins Auge zu fassen. Zudem stehen diese Institutionen vielfach unter Erfolgszwang und müssen ihre Geldgeber mit Projekten zufriedenstellen, bei denen schon nach relativ kurzer Zeit Erfolge ausgewiesen werden können.

Im Selbsthilfeansatz werden partizipative Forschung und Planung, d. h. die aktive Mitarbeit der Zielgruppe, gefordert, wenn z. B. die Durchführbarkeit vorgeschlagener Aktionen eingeschätzt oder Operationen geplant werden sollen. Vielfach ist es aber so, daß Forschungsund Planungsarbeit von den indonesischen NGOs nur von der technischen und wirtschaftlichen Seite her betrachtet werden. Dabei wird außer acht gelassen, daß arme Bevölkerungsschichten weniger durch wirtschaftliche Belange als vielmehr über soziokulturelle Momente miteinander verbunden sind. Um diese identifizieren zu können, sollten die Zielgruppen an den Forschungs- und Planungsprozessen partizipieren.
Unsachgemäße sowie nachlässige Forschungs- und Planungsarbeit verhindert nachträglich auch ein erfolgreiches Monitoring und nachhaltige Evaluationstätigkeiten. Bei Bina Swadaya und LSP sind Forschungs- und Planungsaktivitäten sowie Monitoring und fortlaufende Evaluationen bezüglich ihrer Spar- und Kreditprogramme äußerst erfolgreich. Bei LSP trifft dies auch für ihre Selbsthilfeprogramme und -projekte im städtischen informellen Sektor zu. Schwieriger wird eine Beurteilung jedoch für andere, insbesondere ökonomische Aktivitäten. Problematisch ist in diesem Zusammenhang auch das Fehlen von Kriterien und Bestimmungen, die vorsehen, wann eine Selbsthilfeorganisation in die Unabhängigkeit entlassen werden kann. Dabei muß jedoch dem Umstand Rechnung getragen werden, daß sich der Komplex regierungsunabhängiger Selbsthilfeorganisationen immer noch in einer Aufbauphase befindet und die meisten kaum länger als zehn Jahre existieren. Deshalb haben NGOs noch keine konkreten Konzepte für die Ablösung ihrer Selbsthilfegruppen erarbeitet. Außer Zweifel steht, daß Bina Swadaya und LSP das «institution building» beherrschen. Dies haben sie mit der Gründung und dem Aufbau zahlreicher Selbsthilfegruppen bewiesen. Je länger je mehr stellt sich aber die Frage, ob diese aufgebauten Gruppen auch auf sich allein gestellt lebensfähig sind. Grundsätzlich gilt es zu beachten, daß Entwicklung einen langfristigen und mehrdimensionalen Prozeß darstellt, der verschiedenste Aspekte beinhaltet. Eine zu frühe Ablösung der Selbsthilfegruppe könnte sich eventuell nachteilig auf alle Beteiligten auswirken. Die mit den Selbsthilfeorganisationen aufgebauten langjährigen Beziehungen stellen für die NGOs gewissermaßen ein wertvolles Investitionskapital dar. Vor allem erfolgreiche Programme und Projekte mit Selbsthilfegruppen dienen der Legitimierung der NGOs gegenüber Geldgebern. Solange Selbsthilfegruppen erfolgreich operieren, sollten die Selbsthilfeaktivitäten durch die NGOs diversifiziert und weiterentwickelt werden, denn diese kommen nicht nur den Selbsthilfegruppenmitgliedern und ihren Familien zugute, sondern bewirken erstens direkte Spill-overs auf die unmittelbare Projektumgebung, z. B. durch Verbesserung im Beschäftigungsbereich, in der Infrastruktur usw., sowie zweitens indirekte, nachgelagerte Auswirkungen, z. B. durch Hebung des Wohlstands einer Familie, die ihren Verwandten in anderen Regionen Mittel zufließen läßt. Bis heute ist aber noch sehr wenig darüber bekannt, in welchem Umfang Spill-overs erfolgen und wie sie ihre Umgebung beeinflussen.

Bei den Aus- und Weiterbildungsmethoden fehlt vor allem bei Bina Swadaya die vom Selbsthilfeansatz geforderte Basisnähe und die Synthese traditionellen Wissens der Zielbevölkerung mit von außen eingebrachten Sachkenntnissen. Die Aus- und Weiterbildungskurse werden in einem speziell dafür geschaffenen Trainingszentrum nach der traditionellen "trickle-down"-Methode vermittelt, deren Effekt nachgewiesenermaßen in der Zielgruppe gering ist (BONGARTZ 1987 und VERHAGEN 1987). Eine solche Ausbildungsmethode stellt somit eine einseitige, nicht auf Kommunikation mit der Zielbevölkerung beru- 
hende Vermittlung von Wissen dar, welche auch nicht die Synthese von traditionellem mit "fremdem" Wissen propagiert. Die Methoden von LSP hingegen sind um einiges flexibler und basisnaher. Neben der Kaderschulung und anderen, vor allem auf die Ausbildung von Führungskräften ausgerichteten Kursen bietet LSP ihren Selbsthilfegruppenmitgliedern auch dezentral organisierte, informelle Ausbildungskurse direkt an der Basis an, bei denen die nötigen Fertigkeiten für eine bestimmte Tätigkeit während der Arbeit angelernt und erworben werden. Diese Methode «learning by doing» oder «learning on the job" gehört eigentlich zu den elementarsten Selbsthilfeförderungsinstrumenten und ist in Indonesien bei vielen NGOs bekannt, doch wird sie in den wenigsten Fällen auch wirklich angewandt.

Ressourcenmobilisierung innerhalb der Selbsthilfegruppen dient in Indonesien häufig nur dem Aufbau und der Förderung von Spar- und Kreditaktivitäten (Bina Swadaya) oder von wirtschaftlichen Aktivitäten (LSP), zu deren Zweck von der NGO meistens bloß finanzielle Ressourcen bereitgestellt werden. Doch damit läuft man Gefahr, das Selbsthilfekonzept darauf zu reduzieren, unabhängige Spar- und Kreditsysteme oder wirtschaftlich profitable und in die Marktwirtschaft integrierbare Produktionsbetriebe aufzubauen. Ein aufdiese Weise propagierter Selbsthilfeansatz würde sich in nichts von der Modernisierungsstrategie unterscheiden, die sich unter dem Deckmantel der "Hilfe zur Selbsthilfe» versteckt! Bina Swadaya und LSP sollten ihre Rolle als Selbsthilfeförderungsinstitutionen seriös überdenken, denn einige ihrer vor allem im landwirtschaftlichen Bereich angesiedelten Programme und Projekte sind nichts anderes als Beispiele marktwirtschaftlich orientierter Wirtschaftsförderung.

Die im Selbsthilfeansatz so wichtigen soziokulturellen und partizipatorischen Komponenten sind bei einigen Programmen und Projekten in den Hintergrund getreten. Beide Organisationen, insbesondere Bina Swadaya, sollten deshalb den genannten Komponenten in Zukunft mehr Beachtung schenken, wenn sie Selbsthilfeförderung auf ihr Banner schreiben. Diesbezüglich kommt VERHAGEN (1987) in seiner Studie zu folgender Schlußfolgerung, die gewissermaßen auch für die beiden untersuchten Fallbeispiele Bina Swadaya und LSP zutrifft: "Under present conditions, a lot of the SHPIs' (Selbsthilfeförderungsinstitutionen) attention and energy is devoted to the role they should probably not play, that of a financing agency which acts as an intermediary in the flow of resources from donor agencies to the local SHOs (Selbsthilfegruppen). This leaves them insufficient attention and energy to devote to the role they should play: a catalytic and supportive role in a series of interconnected processes at grassroot level such as mobilization of local resources, organization of the rural poor, and identification and preparation of economic activities which primarily strengthen the latter's economic and social position" (VERHAGEN 1987: S. 141).
(Noch) kein soziopolitischer Wandel von unten mittels Selbsthilfeförderung durch NGOs

Bei einer Annäherung der Selbsthilfeförderungsinstitutionen an staatliche Entwicklungsinstitutionen stellt sich die grundlegende Frage, was damit letztlich erreicht werden soll. Ist die Selbsthilfeförderung das "tatsächliche» und einzige Ziel von Bina Swadaya und LSP, oder ist sie nur ein Mittel für einen anderen Zweck? Die beiden Leiter Bambang Ismawan (Bina Swadaya) und Adi Sasono (LSP) denken und handeln langfristig und politstrategisch. "In their opinion power formation at grassroots level is impossible and they therefore form coalitions with sections of the regime as this is the only means of safeguarding their positions. Some, in having such a if you can't beat them join them' policy, keep an eye on a more long term objective: forming a new power-basis with the support of parts of the present Government" (WITJES 1986: S. 36). Ihre Verdienste im sozialen Entwicklungsbereich könnten vielleicht später ein Sprungbrett für ein politisches Amt abgeben. Was beide anstreben, ist die Veränderung bestehender sozialer, politischer und wirtschaftlicher (Macht-)Strukturen in Indonesien. Als Mittel verwenden sie einerseits die Selbsthilfeförderung armer Bevölkerungsschichten und andererseits die politische Karriere, welche ihnen die nötige Macht zur wirkungsvollen und nachhaltigen Durchsetzung ihrer Ideen in die Hände legen soll.

In Indonesien ist es aufgrund der politischen und soziokulturellen Rahmenbedingungen zur Zeit unmöglich, durch Selbsthilfegruppen eine einflußreiche, soziopolitische Bewegung von unten aufzubauen. Bambang Ismawan und Adi Sasono scheinen sich der Tatsache bewußt zu sein, daß sie geringe Erfolgschancen haben, um mit ihren Organisationen soziopolitischen Wandel einzuleiten. Deshalb mögen sie sich auch dazu entschieden haben, andere Instrumente und Kanäle als diejenigen, welche der Selbsthilfeansatz empfiehlt, zur Verwirklichung ihrer Ziele zu benutzen. Ist man sich dessen bewußt, so werden die Handlungsweisen beider Organisationen transparenter. Der vordergründig propagierte Selbsthilfeansatz beider Organisationen verliert aufgrund der politischen und soziokulturellen Rahmenbedingungen, die soziopolitischen Wandel des Gesellschaftssystems von unten nicht erlauben, an Bedeutung. Der Selbsthilfeansatz versagt in Indonesien aufgrund politischer und soziokultureller Rahmenbedingungen. Darum ist es auch verständlich, daß indonesische Selbsthilfeförderungsinstitutionen von dieser Strategie abkommen und mit anderen Mitteln versuchen, soziopolitische Veränderungsprozesse wie beispielsweise Demokratisierung einzuleiten.

Die Annäherung von Bina Swadaya und LSP an staatliche Entwicklungsinstitutionen durch den Aufbau von Netzwerkorganisationen in Zusammenarbeit mit Regierungsstellen, durch die Übernahme von Teilprojekten aus Regierungsprogrammen sowie durch den Aufbau legaler, staatlich kontrollierter, parapolitischer Massenorganisationen (LSP) oder durch die politische Einfluß- 
nahme in solchen durch Personalunionen (Bina Swadaya) deuten auf Alternativen und Versuche hin, Selbsthilfeideologie in staatliche Strukturen einfließen zu lassen. Dabei sollte man sich der Tatsache bewußt sein, daß die Regierung keinen monolithischen Block darstellt, wie dies manche Studien vorzugeben versuchen. Auch in Regierungskreisen führten Diskussionen zur NGO-Problematik zu kontroversen Ansichten. Einige Ministerien verfolgen durchaus eine NGO-freundliche Politik, die zeitweise schon zu weitgehender Solidarität geführt hat. Von Emil Salim, dem derzeitigen Umweltminister, ist beispielsweise bekannt, daß er den NGOs eine bedeutende Rolle im nationalen Entwicklungsproze $B$ zubilligt. Gewisse Regierungsstellen haben in den vergangenen Jahren einen eigentlichen Sinneswandel bezüglich ihrer Einstellung zu NGOs durchgemacht. So nahm Sek. Kab. (Sekretariat Kabinet $=$ Staatssekretariat $)^{6}$ Anfang der $80 \mathrm{er}$ Jahre NGOs gegenüber noch eine eher ablehnende Haltung ein. Die Ablehnung wandelte sich Mitte der 80er Jahre in "Neutralität» und Anfang der 90er Jahre gar in Solidarität! Zu Beginn der 90er Jahre bewarben sich verschiedene Ministerien und andere Regierungsstellen (Innenministerium, Umweltministerium, Sek. Kab.) um die Übernahme der Koordinationsaufgabe von NGO-Aktivitäten. Gewissen NGOs, insbesondere Bina Swadaya und LSP, wird nachgesagt, daß sie anfangs der 90er Jahre zur "Hausmacht" gewisser progressiv und liberal denkender Politiker, wie beispielsweise des Umweltministers Emil Salim, gehören.

Mit der Unterstützung progressiver, liberaler Regierungskreise ist es durchaus denkbar, daß NGOs einen Beitrag bezüglich soziopolitischer Wandlungsprozesse in Indonesien leisten können. Dazu müssen sie allerdings zu Kompromissen bereit sein, die auch die Aufgabe ihrer ursprünglichen Entwicklungsziele bedeuten können. Eher auf diesem Weg als auf dem des Selbsthilfeansatzes und der soziopolitischen Bewegungsbildung von unten befinden sich auch Bina Swadaya und LSP. Selbst NGOAktivisten bestätigen diesen Trend. Dies kommt z. B in der Aussage von Abdurrahman Wahid, dem Vorsitzenden der Selbsthilfeförderungsinstitution "Bina Desa» und der zweitgrößten muslimischen Massenorganisation Indonesiens "Nahdatul Ulama», zum Ausdruck. Er ist der Ansicht, daß alle "Bottom up»-Entwicklungsstrategien, also auch der Selbsthilfeansatz, in Indonesien purer Mythos und deshalb nicht realisierbar seien: “(...) what NGOs have been doing so far could not be described as carrying out a community-based development but rather as setting up a countermodel of development that originates from the top just like the traditional model. Claims that NGOs have been carrying out community-based development projects are not based on reality; if there are any such programs, they are limited to a few specific cases" (THE JAKARTA POST. 19. Sept. 1989).

Die Tatsache, daß zu Anfang der 90er Jahre weniger als $1 \%$ der gesamten indonesischen Bevölkerung von Selbsthilfeförderungsprogrammen und -projekten erfaßt wurde, zeigt doch, daß das Entwicklungspotential in diesem Bereich noch bei weitem nicht ausgeschöpft ist. Die Fra- ge stellt sich nur, in welche Richtung sich die Selbsthilfeförderungsaktivitäten weiterentwickeln werden. Wird die Annäherung der NGOs an die Regierung mit dem damit verbundenen Verlust der Unabhängigkeit die zukünftige indonesische Realität prägen, oder kommt es zu einer Kehrtwendung und zu einer Autonomiebewegung unter den NGOs? Folgendes Szenario scheint sowohl aus kurz- als auch aus langfristiger Perspektive möglich.

\section{Kurzfristig: \\ unveränderte soziopolitische Rahmenbedingungen}

Solange die soziopolitischen Rahmenbedingungen in Indonesien unverändert bleiben, werden die NGOs ihre Zusammenarbeit mit der Regierung intensivieren, und ihre Rolle als Regierungspartner wird bei der Implementierung von Programmen und Projekten je länger je wichtiger werden. Die Vorbildfunktion, welche Entwicklungsmodelle von NGOs gegenüber staatlichen Entwicklungsstellen haben, wird in den kommenden Jahren noch ausgebaut werden, da sich die Regierung bewußt geworden ist, daß ihre eigenen Programme und Projekte davon profitieren können. Die eigentliche Selbsthilfeförderung wird dabei aber einen immer geringeren Anteil innerhalb von NGO-Programmen einnehmen. Auch wenn die staatlichen Entwicklungsstellen bei der Zusammenarbeit mit NGOs diesen in einem gewissen $\mathrm{Ma} ß$ entgegenkommen, werden sich die NGOs den Regierungsprogrammen stärker anpassen müssen als umgekehrt. Denn nicht Selbsthilfeförderungsinstitutionen vergeben Projektimplementierungen, sondern Regierungsstellen.

Die großen NGOs werden einerseits ihre soziopolitischen Maximen vermehrt zugunsten dienstleistungsbezogener Entwicklungsaufgaben einbüßen. Viele NGOs werden ihre Unabhängigkeit ganz oder zumindest teilweise verlieren, da sie durch die Übernahme von staatlichen Regieaufträgen vermehrt ins staatliche Entwicklungsnetz eingebunden werden. Dadurch besteht aber andererseits die Möglichkeit, den Staat zu beeinflussen, indem man Selbsthilfeförderungsideologie in Regierungsprojekte einfließen lassen und die gewünschte Entwicklungswirkung multiplizieren kann. Eine NGO muß also ihre ursprünglichen Entwicklungsziele und ihre Ideologie nicht unbedingt in Frage stellen, wenn sie intensiver mit Regierungsstellen zusammenarbeitet. Aber in der Regel ist dieser Trend vielfach festzustellen. Dieses Szenario zeichnet sich bei Bina Swadaya Anfang der $90 e r$ Jahre ab. NGOs, die ihre Unabhängigkeit zu bewahren bestrebt bzw. nur begrenzt mit Regierungsstellen zusammenzuarbeiten gewillt sind, werden sich zusammen mit den Geberorganisationen auf alternative Projekttypen konzentrieren, die mit den Zielen der Selbsthilfeförderung nur noch begrenzt in Verbindung gebracht werden können. So wird zur Zeit im Zusammenhang mit dem Ausbau staatlich unabhängiger Kreditsysteme eine neuartige Form von Projekten erprobt. Bei diesen werden Selbsthilfeförderungsinstitutionen von ausländi- 
schen Geberorganisationen als Geschäfts- und Vermittlungsstellen für Bankgeschäfte gesucht, wobei die ausländischen Geber in den Hintergrund treten und nur noch mittels Bankbürgschaften an inländische Banken indirekte Entwicklungshilfe leisten. Somit entsteht ein Entwicklungsdreieck zwischen ausländischen Geberorganisationen (die mittels Bankbürgschaften die Garantie für allfällige Risiken bei der Kreditvergabe durch die inländischen Banken übernehmen), inländischen Banken (welche die Kredite zur Verfügung stellen) und den Selbsthilfeförderungsinstitutionen (die als Informations- und Koordinationsstellen zwischen den Banken, den ausländischen Gebern und den Kreditnehmern fungieren). Ein solches System wird z. B. in den Philippinen bereits erfolgreich implementiert. Auch Bina Swadaya hat unter der Ägide der deutschen Regierung und der indonesischen Nationalbank (Bank Indonesia) in diesem Bereich ein Pilotprojekt laufen. Dieser Projekttyp ermöglicht den Aufbau einer neuen kommerziellen Entwicklungszusammenarbeit im Bereich der Basisentwicklung. Dabei sollen bereits vorhandene, lokale Strukturen benützt und verbessert werden und zugleich Entwicklungsgelder effizienter als bisher direkt an die Basis gelangen. Diese innovativen Projekttypen werden heute fälschlicherweise unter dem Namen des Selbsthilfeansatzes geführt und gefördert. Doch ist ein Aufbau von Kreditsystemen mit den Hauptzielen der Selbsthilfeförderung, nämlich der Bewußtseinsbildung marginalisierter Bevölkerungsschichten zum Aufbau einer Wandel induzierenden, soziopolitischen Bewegung von unten, kongruent?

Kurzfristig wird diese Entwicklung in Indonesien nicht zu umgehen sein, denn der Selbsthilfeansatz, wie er von VERHAGEN (1987) propagiert wird, hat klare politische Zielsetzungen, die aufgrund der vorherrschenden politischen und soziokulturellen Rahmenbedingungen nicht realisierbar sind.

\section{Langfristig: politische Öffnung}

Eine politische Öffnung des Landes hin zu demokratischen Prinzipien und der Abbau nach wie vor vorhandener Konfliktpotentiale in religiösen, ethnischen, bevölkerungspolitischen und anderen Bereichen gehören zu den Voraussetzungen, damit der Selbsthilfeansatz seinen soziopolitischen Ansprüchen gerecht werden und somit erfolgreich implementiert werden kann. Langfristig wird sich eine schon heute feststellbare Dichotomisierung der NGOs in zwei Gruppen vollziehen, ähnlich wie dies ELDRIDGE (1989: S. 11) in seinem Modell zu erklären versucht. NGOs, die sich zu einer Zusammenarbeit mit der Regierung entschließen, werden als unpolitische Dienstleistungsorganisationen ihr Geld verdienen. Gegen die Regierung opponierende NGOs bilden den anderen Pol. Bislang werden sie von der Regierung unterdrückt und haben keinen signifikanten Einfluß auf die politischen Handlungsweisen der Regierung. Sollte aber eine politische Liberalisierung erfolgen, bestünde durchaus eine Chance für die Realisierbarkeit der soziopolitischen
Zielsetzungen des Selbsthilfeansatzes. Die Selbsthilfeförderungsinstitutionen könnten dabei zu einer wirksamen und treibenden Kraft des politischen und soziokulturellen Entwicklungsprozesses in Indonesien heranwachsen, indem sie als Massenorganisationen mitbestimmen und die Wünsche und Bedürfnisse der ärmeren Bevölkerungsschichten in die nationale Politik einbringen könnten.

An eine politische Öffnung wäre allenfalls in der NachSuharto-Ära zu denken. Progressiv und alternativ denkende politische Kreise, denen die meisten der NGOFührer sowie einige maßgebende Politiker angehören, arbeiten bereits intensiv an der Gestaltung der zukünftigen politischen Landschaft. Ob eine solche Änderung der politischen Rahmenbedingungen noch vor der Jahrtausendwende erfolgen wird, muß abgewartet werden.

\section{Anmerkungen}

'Im folgenden wird die Abkürzung "NGO" dem Begriff "Selbsthilfeförderungsinstitution" gleichgestellt.

${ }^{2}$ Die Umrechnungen fußen auf den Kursen von 1988.

${ }^{3}$ Bsp. Bina Swadaya: Im Trainingszentrum von Bina Swadaya (PUSDIKLAT) in Cimanggis (in der Nähe von Bogor) werden regelmäßig folgende Trainings- und Weiterbildungskurse für Selbsthilfegruppenmitglieder angeboten:

- Vermittlung von Bina Swadayas Selbsthilfegruppenprinzipien (Organisationsstrukturen und Aufbau einer Selbsthilfegruppe usw.) an alle Mitglieder jeder neu gegründeten Gruppe;

- Vermittlung von Führungskenntnissen an ausgewählte Mitglieder der Gruppen;

- Buchhaltung und Administration;

- ökonomisches Grundwissen und Kapitalbildung;

- Motivation zu privatem Unternehmertum für Kleinst- und Familienbetriebe;

- technische Ausbildung;

- Evaluations- und Planungsmethoden;

- Verwaltung eines Kleinbetriebes;

- Marktanalysen für Kleinbetriebe.

${ }^{4}$ Diese Doppelfunktion (dwi-fungsi) der indonesischen Streitkräfte wird mit der sowohl militärischen als auch politischen Beteiligung der Widerstandsbewegung im Kampf um die Unabhängigkeit legitimiert. Offiziell wird diese Verbindung SOSPOL (sosial dan politik) genannt und ist zum zentralen Begriff des militärischen Selbstverständnisses geworden.

5 "Gotong royong" kann als "uneigennützige, auf Gegenseitigkeit beruhende Hilfe" übersetzt werden. WANDELT definiert den Begriff folgendermaßen: "Gotong royong ist die informelle und temporäre soziale Arbeitsteilung innerhalb der Dorfgemeinschaft, die zur Lösung gemeinschaftlicher Aufgaben und Probleme und für das Wohl aller Beteiligten erstellt wird. Es betrifft i. d. R. regelmäßig auftretende Routineaufgaben wie das Einbringen der Ernte im landwirtschaftlichen Zyklus, Bau und Pflege von Gemeinschaftseinrichtungen, aber auch Katastrophenhilfe. Dem Gotong royong geht ein musyawarah voraus, auf dem das Problem behandelt, eine Lösungsstrategie erarbeitet und ein Arbeitsplan einschließlich der Verteilung der Aufgaben von allen gemeinsam beschlossen wird. (...) Gotong royong [ist] ein Symbol der Solidarität der Individuen mit ihrer sozialen (Dorf-)Gemeinschaft (...)." (WANDELT 1989: S. 252). 
${ }^{6}$ Sekretariat zur Koordination der Tätigkeiten des Präsidenten u. a. auch oberste Prüfungsinstanz für Projektbewilligungen und -verträge ausländischer Entwicklungsinstitutionen, die mit indonesischen NGOs zusammenarbeiten.

\section{Literaturverzeichnis}

BIJLMER, J./REURINK, M. (1986): Local Leadership in Urban Neighbourhoods in Java: From Ideology to Reality, in Ufford 1986, S. 139-160.

BINA SWADAYA (1986): Agency Profile, Jakarta.

BONGARTZ, H. (1987): Prospects and Limitations for SelfHelp Organisations in the Development Process of Rural Java, unveröffentlichte Dissertation an der Universität Bielefeld.

BONGARTZ, H./KUSUMAHADI, M. (1988): Some Alternatives for Bilateral Cooperation involving NGOs in the Field of Small Productive Enterprise Development in the Informal Sector in Indonesia, unveröffentlichte Studie, Jakarta und Bielefeld.

ELDRIDGE, P.(1989): NGOs in Indonesia: Popular Movement or Arm of Government?, Working Paper 55, Centre of Southeast Asian Studies, Monash University, Clayton, Australien.

GUINAND, Y. (1991): Möglichkeiten und Grenzen der Selbsthilfeförderung in Indonesien. Dargestellt an den beiden regierungsunabhängigen Organisationen Bina Swadaya und Lembaga Studi Pembangunan, unveröffentlichte Diplomarbeit am Geographischen Institut der Universität Zürich.

IFDA (1989): Indonesia: Usaha Bersama Arisan Manjung. In: Ifda-Dossier 72, S. 15-23.
ISMAWAN, B. (1987): NGO Lessons from Indonesia. Unveröffentlichtes Paper, Jakarta.

JAKARTA POST (1989): NGOs Development Strategy Remains Myth: Abdurrahman, 19. September.

KORTEN, D. C. (1989): Voluntary Organizations and the Challenge of Sustainable Development, Institute for Development Research, Manila.

KÖTTER, H./JUNGHANS, K.H./ROEDER, R. O.G. (Hrsg.) (1979): Indonesien, Horst Erdmann Verlag, Tübingen und Basel.

KUSUMAHADI, M. (1987): Basic Information on the Various Perspectives of the Urban Informal Sector in Indonesia. Unveröffentlichte Studie für die Friedrich Ebert Stiftung (FES), Jakarta.

ROEDER, R. O.G. (1979): Zur Identität der indonesischen Gesellschaft, in Kötter/Junghans/Roeder 1979, S. 181-198.

SALIM, E. (1984): Common Aims, Different Approaches, in Prisma Nr. 28, S. 70-72, Jakarta.

SASONO, A. (1988): People's Economy, South-East Asian Forum for Development Alternatives (SEAFDA) (Hrsg.), Jakarta.

VERHAGEN, K. (1987): Self-help Promotion, a challenge to the NGO-Community, Studie im Auftrag von CEBEMO und dem Royal Tropical Institute, Amsterdam.

WANDELT, I. (1989): Der Weg zum Pancasila-Menschen. In: Europäische Hochschulschriften, Peter Lang Verlag, Frankfurt am Main, Bern, New York, Paris.

WITJES, B. (1986): The Indonesian Law on Social Organisations. A Study of the Sociopolitical Context and the Consequences for Indonesian and Foreign NGOs, unveröffentlichte Studie im Auftrag von NOVIB, Nijmegen, Niederlande. 